\title{
Compact Water Vapor Exchanger for Regenerative Life Support Systems
}

\author{
Abstract submitted for the $43^{\text {rd }}$ International Conference on Environmental Systems \\ Session: ICES408: Regenerable Life Support Processes and Systems \\ (Please let me know if you agree with this session. Other possible sessions are ICES200: Physico-Chemical \\ Processes: Air and Water, and ICES302: Physio-Chemical Life Support Process Development). \\ Michael G. Izenson and Weibo Chen \\ Creare Inc., Hanover, New Hampshire, 03755 \\ Molly Anderson \\ NASA Lyndon B. Johnson Space Center, Houston, Texas, 77062 \\ Edward Hodgson \\ United Technologies Corporation Aerospace Systems, Windsor Locks, Connecticut
}

Thermal and environmental control systems for future exploration spacecraft must meet challenging requirements for efficient operation and conservation of resources. Regenerative $\mathrm{CO}_{2}$ removal systems are attractive for these missions because they do not use consumable $\mathrm{CO}_{2}$ absorbers. However, these systems also absorb and vent water to space along with carbon dioxide. This paper describes an innovative device designed to minimize water lost from regenerative $\mathrm{CO}_{2}$ control systems. Design studies and proof-of-concept testing have shown the feasibility of a compact, efficient membrane water vapor exchanger (WVX) that will conserve water while meeting challenging requirements for operation on future spacecraft. Compared to conventional WVX designs, the innovative membrane WVX described here has the potential for high water recovery efficiency, compact size, and very low pressure losses. The key innovation is a method for maintaining highly uniform flow channels in a WVX core built from water-permeable membranes. The proof-of-concept WVX incorporates all the key design features of a prototypical unit, except that it is relatively small scale (1/23 relative to a unit sized for a crew of six) and some components were fabricated using non-prototypical methods. The proof-of-concept WVX achieved over $90 \%$ water recovery efficiency in a compact core in good agreement with analysis models. Furthermore the overall pressure drop is very small (less than $0.5 \mathrm{in}$. $\mathrm{H}_{2} \mathrm{O}$, total for both flow streams) and meets requirements for service in environmental control and life support systems on future spacecraft. These results show that the WVX provides very uniform flow through flow channels for both the humid and dry streams. Measurements also show that $\mathrm{CO}_{2}$ diffusion through the water-permeable membranes will have negligible effect on the $\mathrm{CO}_{2}$ partial pressure in the spacecraft atmosphere. 